\title{
Modellierungskonzept für MOS Varaktoren zur Minimierung der AM-FM Konversion in VCOs
}

\author{
T. Peikert, J.-K. Bremer, and W. Mathis \\ Leibniz Universität Hannover, Institut für Theoretische Elektrotechnik, Appelstr. 9A, 30167 Hannover, Germany
}

Zusammenfassung. In dieser Arbeit wird ein analytisches Simulationsmodell für MOS Varaktoren zur Entwurfsunterstützung von integrierten CMOS LC-Tank VCOSchaltungen präsentiert. Das analytische Simulationsmodell wurde auf Basis des EKV-Transistormodells implementiert und beinhaltet ausschließlich Design- und Prozessparameter für die Berechnung der Varaktorkapazität. Dieses Simulationsmodell ermöglicht es, die verwendeten Varaktoren im Vorfeld des VCO-Entwurfs zu dimensionieren, die effektive Großsignalkapazität in Abhängigkeit des Ausgangssignals $\mathrm{zu}$ berechnen und einzelne Eigenschaften der Varaktoren, wie z.B. das AM-FM Konversionsverhalten zu optimieren. Die Gültigkeit des vorgestellten analytischen Simulationsmodells zur Beschreibung der Varaktorkapazität in CMOS LC-Tank VCOs, wird anhand von Spectre (Cadence) Simulationen auf Basis eines $0.25 \mu \mathrm{m}$ CMOS Prozesses der Firma IHP (SGB25) und eines 0.35 $\mu$ m CMOS Prozesses der Firma AMS (C35) verifiziert.

Abstract. In this work an analytical simulation model for MOS varactors, that can be used in a systematically VCO design flow, is presented. The simulation model is based on the EKV transistor model and includes only design and process parameters of the used CMOS technology. The proposed simulation model allows calculating the required design parameters and the effective large signal capacitance of the varactors incorporated into the VCO as a function of the output signal of the VCO. Based on the expression for the effective large signal capacitance it is possible to optimize the AMFM conversion behavior of the used varactors. The validity and accuracy of the simulation model is verified by Spectre simulations which are based on a $0.25 \mu \mathrm{m}$ CMOS process (SGB25) from the company IHP and a $0.35 \mu \mathrm{m}$ CMOS process (C35) from the company AMS. The simulation results show a good accordance in all transistor operating regions for NMOS varactors as well as PMOS varactors.

Correspondence to: J.-K. Bremer (bremer@tet.uni-hannover.de)

\section{Einleitung}

Um die steigenden Anforderungen in den Spezifikationen für Breitbandanwendungen und Multiband Receiver zu erfüllen, werden integrierte CMOS VCOs, die einen hohen Abstimmbereich besitzen, benötigt. Aufgrund der hohen Verbreitung von LC-Tank VCOs (Herhenson et al., 1999) liegt der Fokus dieser Arbeit auf dieser Oszillatorarchitektur (vgl. Abb. 1). Um die geforderte hohe Abstimmbarkeit zu erreichen, werden häufig MOS Varaktoren oder Arrays von MOS Varaktoren eingesetzt (Buonomo, 2008). Diese besitzen aufgrund ihrer Verschaltung im VCO den Nachteil, dass die effektive Varaktorkapazität nicht nur von der Steuerspannung, sondern auch von der Oszillatoramplitude abhängt. In der LC-Tank VCO Topologie liegt die Ausgangsamplitude direkt über den Gateanschlüssen der verwendeten MOS Varaktoren. Das hat zur Folge, dass sich die sich einstellende effektive Kapazität der Varaktoren periodisch mit dem Verlauf des Ausgangssignals ändert. Dieser Effekt bewirkt eine direkte Abhängigkeit der Varaktorkapazität von der Tankamplitude. Kleine Amplitudenschwankungen, z.B. aufgrund von Rauschen, führen zu einer Modulierung der effektiven Kapazität und damit zu einer Veränderung der Ausgangsfrequenz des VCOs. Dieser Vorgang wird AM-FM Konversion genannt (Hegazi und Abidi, 2003). Um die Phasenrauscheigenschaften des VCOs zu optimieren, muss dieser Effekt beim Schaltungsentwurf berücksichtigt werden. In dieser Arbeit wird ein analytisches Simulationsmodell für Varaktoren vorgestellt, mit dem im Vorfeld des Entwurfs, ohne Zuhilfenahme eines professionellen EDA-Tools, die CV-Charakteristik der verwendeten Varaktoren bestimmt werden kann. Dieses Vorgehen erlaubt es eine geeignete Approximation der Varaktordimensionen, die die Spezifikationen erfüllen, zu erhalten. Auf Basis des analytischen Varaktorsimulationsmodells lassen sich weiterhin einzelne Eigenschaften der Schaltung optimieren, wie zum Beispiel das AM-FM Konversionsverhalten der Varaktoren. Um ein solches Simulationsmodell zu implementieren, wird ein Transistormodell benötigt, mit dem die Kapazität in kontinuierlicher Form über alle Betriebsbereiche

Published by Copernicus Publications on behalf of the URSI Landesausschuss in der Bundesrepublik Deutschland e.V. 


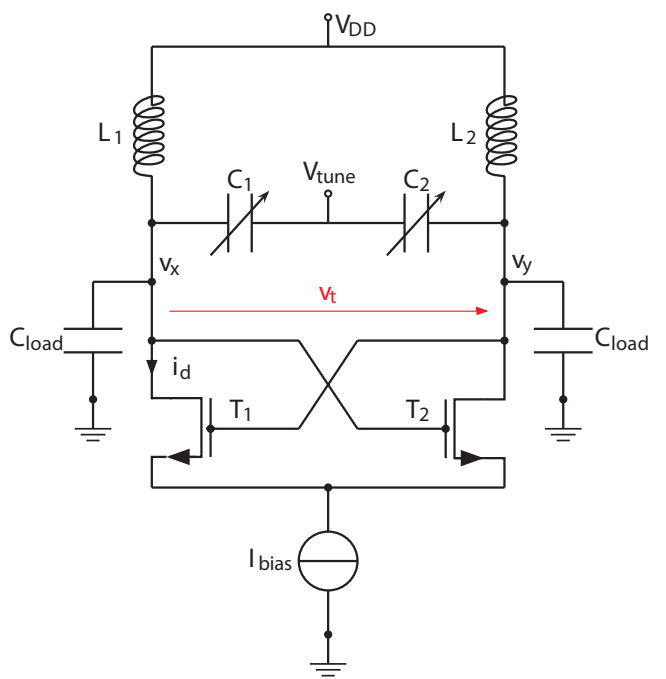

Abb. 1. Standard CMOS LC-Tank VCO Architektur.

beschrieben wird. Viele weit verbreitete Transistormodelle, wie z.B. das BSIM Modell, verwenden eine abschnittsweise Beschreibung der Kapazitätscharakteristik und passen diese über komplexe mathematische Operationen aneinander an. Deshalb wurde als Basis des analytischen Simulationsmodells für die Spannungs-Kapazitätscharakteristik von MOS Varaktoren das EKV-Transistormodell (Enz und Vittoz, 2006) gewählt. Die Gültigkeit und Genauigkeit des vorgestellten Varaktorsimulationsmodells wird durch Simulationen auf Basis eines 0.25 $\mu \mathrm{m}$ CMOS Prozesses der Firma IHP und eines $0.35 \mu \mathrm{m}$ CMOS Prozesses der Firma AMS verifiziert.

\section{Modellierung der Varaktorkapazität}

Die Berechnung der Varaktorkapazität beschränkt sich in dieser Arbeit auf sogenannte InversionsmodeVaraktoren, da diese im Gegensatz zu den sogenannten Akkumulationsmode-Varaktoren aus Standard MOSFETZellen aufgebaut werden. Bei NMOS InversionsmodeVaraktoren wird das Substrat mit Masse verbunden (siehe Abb. 2). Im Falle eines PMOS Inversionsmode-Varaktors wird das Substrat auf das höchste verfügbare Potential (Versorgungsspannung) gelegt (siehe Abb. 3). Zur Implementierung des Simulationsmodells für InversionsmodeVaraktoren, wie bereits in der Einleitung angesprochen, auf das EKV-Transistormodell zurückgegriffen (Bucher et al., 1996). Diese Wahl wurde aufgrund der kontinuierlichen Beschreibung der MOS-Transistoren im Arbeitsbereich von schwacher bis starker Inversion und der geringeren Komplexität des EKV-Modells im Vergleich zu anderen Transistormodellen getroffen. Das EKV-Modell verwendet als Basis die sogenannte Pinch-Off Spannung (Stefanovic und Kaya, 2008), welche folgendermaßen definiert ist

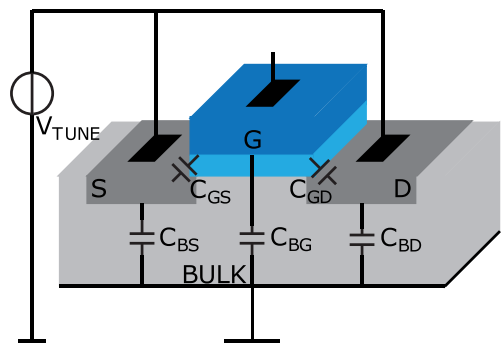

Abb. 2. Intrinsische Kapazitäten, NMOS Inversionsmode-Varaktor.

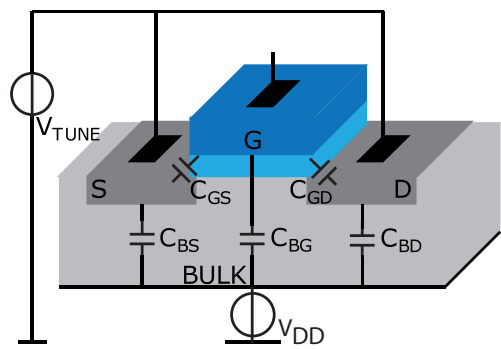

Abb. 3. Intrinsische Kapazitäten, PMOS Inversionsmode-Varaktor.

$V_{\mathrm{P}}=V_{\mathrm{G}}{ }^{\prime}-\mathrm{PHI}-\gamma \cdot\left(\sqrt{V_{\mathrm{G}}{ }^{\prime}+\left(\frac{\gamma}{2}\right)^{2}}-\frac{\gamma}{2}\right)$.

Dabei bezeichnet $V_{\mathrm{G}}{ }^{\prime}$ die effektiven Gatespannung

$V_{\mathrm{G}}{ }^{\prime}=V_{\mathrm{G}}-\mathrm{VTH} 0+\mathrm{PHI}+\gamma \sqrt{\mathrm{PHI}}$,

in der VTH0 die Schwellwertspannung darstellt. Die PinchOff Spannung berechnet sich aus der angelegten Gatespannung $V_{\mathrm{G}}$, dem Fermipotential

$\mathrm{PHI}=2 V_{t}(\mathrm{TNOM}) \cdot \ln \left(\frac{\mathrm{NSUB} \cdot 10^{6}}{n_{i}(\mathrm{TNOM})}\right)$,

mit der Raumtemperatur TNOM, der Oberflächen Dotierkonzentration NSUB, der intrinsische Ladungsträgerdichte $n_{i}$ und dem Substratsteuerfaktor

$\gamma=\frac{\sqrt{2 q \varepsilon_{\mathrm{Si}} \cdot\left(\mathrm{NSUB} \times 10^{6}\right)}}{\operatorname{COX}}$.

Der Substratsteuerfaktor wird durch die Oxidkapazität COX bestimmt, die sich aus der Division von Oxidelektrizitätskonstanten und Oxiddicke ergibt. Des weiteren enthält (4) die Elementarladung $q$ und die Dielektrizitätskonstante für Silizium $\epsilon_{\mathrm{Si}}$. Weiterhin wird für die Bestimmung der Kapazität der sogenannte Slopefaktor,

$n_{q}=1+\frac{\gamma}{2 \sqrt{\mathrm{PHI}+\frac{V_{\mathrm{P}}}{2}}}$, 
benötigt, der sich aus dem Fermipotential und der Pinch-Off Spannung ergibt. Die Pinch-Off Spannung und der Slopefaktor stellen im EKV-Transistormodell die Basis zur Berechnung der intrinsischen Kapazitäten des Varaktors dar. Für die Berechnung der intrinsischen Kapazitäten wird der Drainstrom benötigt. Dieser wird im EKV-Modell (Bucher et al., 1997) durch einen Vorwärts- und einen Rückwärtsstrom

$i_{\text {for }}=\ln ^{2}\left(1+\exp \left(\frac{V_{\mathrm{P}}-V_{\mathrm{S}}}{2 V_{\text {Tune }}}\right)\right)$

und

$i_{\text {rev }}=\ln ^{2}\left(1+\exp \left(\frac{V_{\mathrm{P}}-V_{\mathrm{D}}}{2 V_{\text {Tune }}}\right)\right)$.

definiert. Diese Aufteilung entsteht durch den einheitlichen Bezug der Potentiale auf das Substrat. Aus dem Vorwärtsund Rückwärtsstrom werden die skalierten normierten Ströme $x_{\text {for }}$ und $x_{\text {rev }}$ bestimmt, die sich über $x_{\text {for }}=\sqrt{1 / 4+i_{\text {for }}}$ und $x_{\text {rev }}=\sqrt{1 / 4+i_{\text {rev }}}$ berechnet werden können. Damit lassen sich die interpolierten intrinsischen Teilkapazitäten des Varaktors (Bucher et al., 1997) wie folgt beschreiben

$C_{\mathrm{GS}}=\frac{2}{3} \cdot C_{\mathrm{ox}} \cdot\left(1-\frac{x_{\mathrm{rev}}^{2}+x_{\mathrm{rev}}+\frac{1}{2} x_{\mathrm{for}}}{\left(x_{\mathrm{for}}+x_{\mathrm{rev}}\right)^{2}}\right)$,

$C_{\mathrm{GD}}=\frac{2}{3} \cdot C_{\mathrm{ox}} \cdot\left(1-\frac{x_{\mathrm{for}}^{2}+x_{\mathrm{for}}+\frac{1}{2} x_{\mathrm{rev}}}{\left(x_{\mathrm{for}}+x_{\mathrm{rev}}\right)^{2}}\right)$,

$C_{\mathrm{GB}}=C_{\mathrm{ox}} \cdot\left(\frac{n_{q}-1}{n_{q}}\right) \cdot\left(1-c_{\mathrm{gs}}-c_{\mathrm{gd}}\right)$,

$C_{\mathrm{SB}}=\left(n_{q}-1\right) \cdot C_{\mathrm{GS}}$ und $C_{\mathrm{DB}}=\left(n_{q}-1\right) \cdot C_{G D}$.

Eine andere Variante zur Berechnung der intrinsischen Kapazitäten des Varaktors im ladungsbasierten EKV-Modell ist die Berechnung über die differentiellen Ladungen. Die Drain-, Source-, Gate-, Bulk und Inversionsladungen können über folgende Formeln berechnet werden (Enz und Vittoz, 2006)

$$
\begin{aligned}
& q_{\mathrm{D}}=-n_{q}\left(\frac{4}{15} \cdot \frac{3 x_{r}^{3}+6 x_{r}^{2} x_{f}+4 x_{r} x_{f}^{2}+2 x_{f}^{3}}{\left(x_{f}+x_{r}\right)^{2}}-\frac{1}{2}\right), \\
& q_{\mathrm{S}}=-n_{q}\left(\frac{4}{15} \cdot \frac{3 x_{f}^{3}+6 x_{f}^{2} x_{r}+4 x_{f} x_{r}^{2}+2 x_{r}^{3}}{\left(x_{f}+x_{r}\right)^{2}}-\frac{1}{2}\right), \\
& q_{\mathrm{I}}=q_{\mathrm{S}}+q_{\mathrm{D}}=-n_{q} \cdot\left(\frac{4}{3} \cdot \frac{x_{f}^{2}+x_{f} x_{r}+x_{r}^{2}}{x_{f}+x_{r}}-1\right),
\end{aligned}
$$

$$
q_{\mathrm{B}}=\left(-\gamma \cdot \sqrt{V_{\mathrm{P}}+\mathrm{PHI}+10^{-6}}\right) \cdot \frac{1}{V_{t}}-\left(\frac{n_{q}-1}{n_{q}}\right) \cdot q_{\mathrm{I}} .
$$

Die Gateladung $q_{\mathrm{G}}$ lässt sich über die Beziehung $q_{\mathrm{G}}=-q_{\mathrm{I}}-$ $q_{\mathrm{OX}}-q_{\mathrm{B}}$ berechnen. Dabei bezeichnet $q_{\mathrm{B}}$ die Bulkladung, $q_{\mathrm{I}}$ die Inversionsladung und $q_{\mathrm{OX}}$ die feste Oxidladung, die zu null angenommen wird. Mit diesen differentiellen intrinsischen Ladungen werden über den Zusammenhang

$Q_{(\mathrm{I}, \mathrm{B}, \mathrm{D}, \mathrm{S}, \mathrm{G})}=C_{\mathrm{OX}} \cdot V_{t} \cdot q_{(\mathrm{I}, \mathrm{B}, \mathrm{D}, \mathrm{S}, \mathrm{G})}$

die einzelnen Ladungen berechnet. Die jeweilige Teilkapazität (siehe Abb. 2 und 3) wird durch die Differentiation nach der jeweiligen Spannung über folgenden Ausdruck berechnet

$$
C_{x y}= \pm \frac{\partial}{\partial V_{y}}\left(Q_{x}\right) ; x, y=\mathrm{G}, \mathrm{D}, \mathrm{S}, \mathrm{B} .
$$

Mit den Gleichungen (8) bis (11) und (17) lässt sich die Varaktorgesamtkapazität durch die Parallelschaltung der jeweiligen Teilkapazitäten in Abhängigkeit der angelegten Gatespannung berechnen. Aufgrund ihrer Verschaltung auf ein festes Potential können die Source-Bulk- und Drain-BulkKapazität vernachlässigt werden. Somit erhält man die Gesamtkapazität des Varaktors durch Addition der folgenden intrinsischen Teilkapazitäten

$C_{\mathrm{Ges}}=C_{\mathrm{GB}}+C_{\mathrm{GD}}+C_{\mathrm{GS}}$.

Die Abb. 4 bis 7 zeigen Simulationsergebnisse für die CV-Charakteristik für NMOS und PMOS InversionsmodeVaraktoren, die mit Hilfe einer Implementierung des vorgestellten Simulationsmodells in dem Computeralgebrasystem Maple erzeugt wurden. Abbildung 4 zeigt die erzielten Simulationsergebnisse für einen 0,35 $\mu \mathrm{m}$ CMOS Prozess der Firma AMS (C35) für einen NMOS Inversionsmode-Varaktor mit einer Weite von $100 \mu \mathrm{m}$, einer Länge von $500 \mathrm{~nm}$ und einer Steuerspannung von $V_{\text {Tune }}=1,5 \mathrm{~V}$. Diese werden mit $\mathrm{Si}$ mulationsergebnissen verglichen, die durch eine Meßschaltung in Spectre erzeugt wurden. Abbildung 5 stellt die Simulationsergebnisse für einen $0,25 \mu \mathrm{m}$ CMOS Halbleiterprozesses der Firma IHP (SGB25V) dar. In Abb. 6 und 7 sind die äquivalenten Simulationsergebnisse für einen PMOS Varaktor mit einer Weite von $100 \mu \mathrm{m}$, einer Länge von $500 \mathrm{~nm}$ und einer Steuerspannung von $V_{\text {Tune }}=0 \mathrm{~V}$ zusammengefasst. Die beiden vorgestellten unterschiedlichen Ansätze zur Modellierung der Varaktorkapazität (strombasiert oder über die differentiellen Ladungen) zeigen in allen Bereichen sowohl für NMOS-Varaktoren als auch PMOS-Varaktoren eine gute Übereinstimmung der CV-Charakteristik mit den Simulationsergebnissen aus Spectre (Cadence).

Beide Modelle spiegeln den CV-Verlauf in Steigung, Verschiebung durch die Steuerspannung und minimale und maximale Kapazität gut wieder. Das komplexere Modell über 


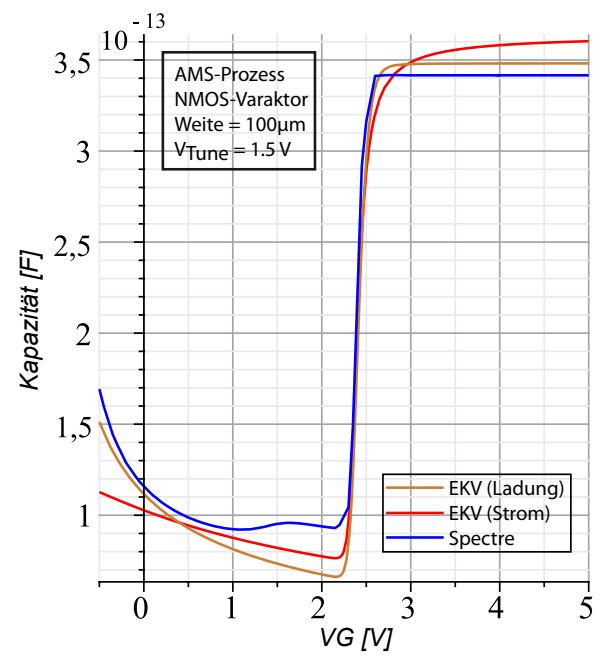

Abb. 4. Simulationsergebnisse für die CV-Charakteristik eines NMOS Inversionsmode-Varaktors (AMS C35).

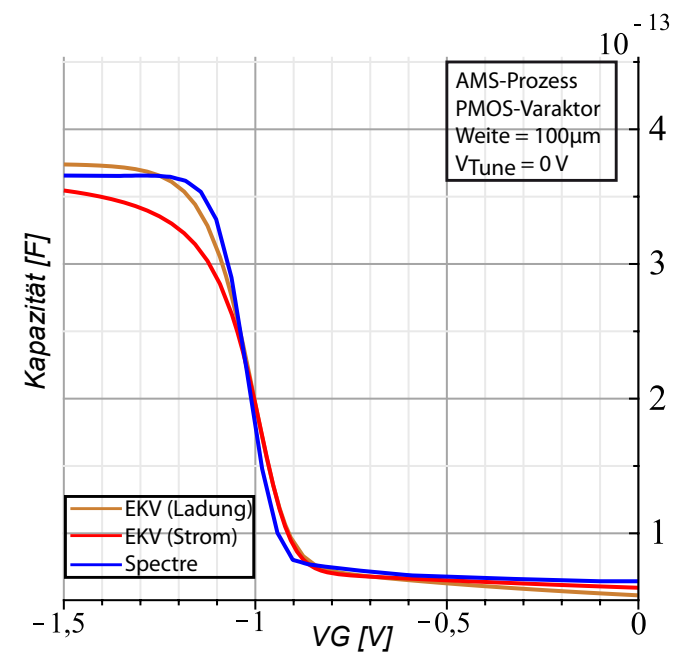

Abb. 5. Simulationsergebnisse für die CV-Charakteristik eines PMOS Inversionsmode-Varaktors (AMS C35).

die differentiellen Ladungen (braun) zeigt gegenüber dem strombasierten Modell (rot) eine bessere Übereinstimmung mit den Spectre (Cadence) Simulationsergebnissen. Zur Verwendung im Vorfeld des VCO Entwurfs ist das weniger exakte strombasierte Modell, aufgrund der geringeren Komplexität und daraus folgenden einfacheren Handhabbarkeit, dem auf den differentiellen Ladungen basierenden Modell vorzuziehen.

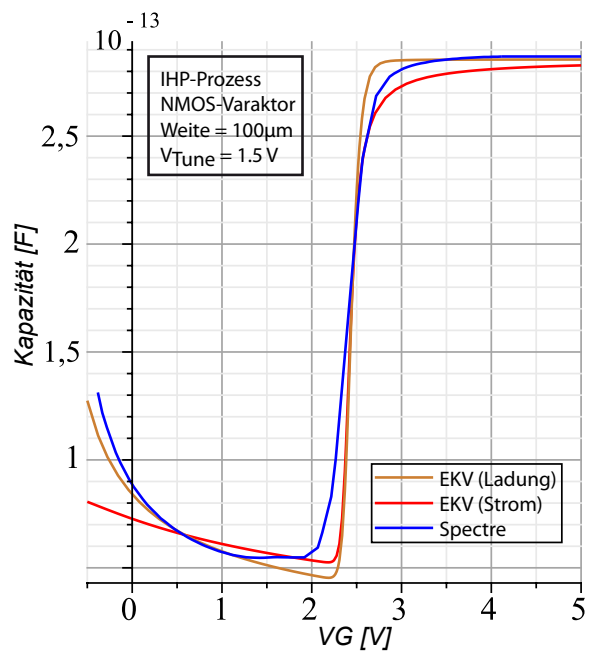

Abb. 6. Simulationsergebnisse für die CV-Charakteristik eines NMOS Inversionsmode-Varaktors (IHP SGB25).

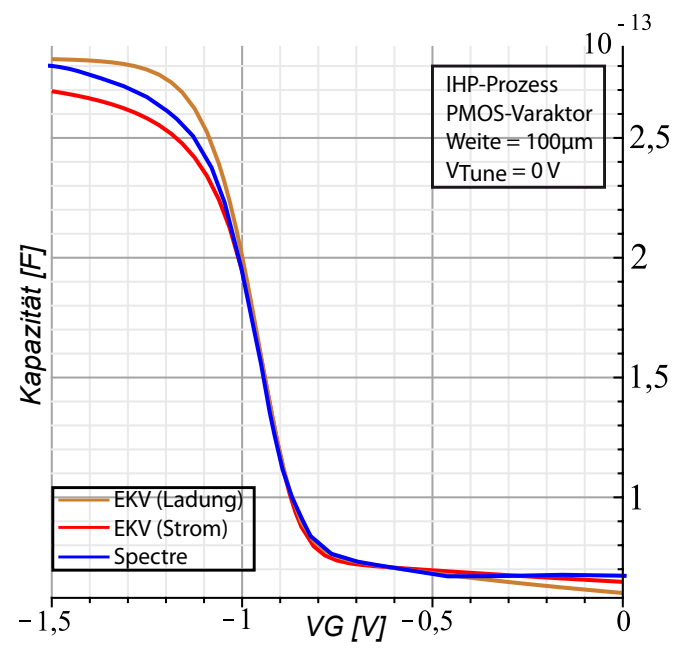

Abb. 7. Simulationsergebnisse für die CV-Charakteristik eines PMOS Inversionsmode-Varaktors (IHP SGB25).

\section{Anwendung des Simulationsmodells}

Für das in Abschnitt 2 vorgestellte analytische Varaktorsimulationsmodell sollen im folgenden Abschnitt einige Anwendungsmöglichkeiten aufgezeigt werden.

\subsection{Unterschiedliche Varaktorverschaltungen}

Das vorgestellte analytische Simulationsmodell ist nicht auf die Beschreibung der CV-Charakteristik von back-toback verschalteten Inversionsmode-MOS-Varaktoren in einer Standard LC-Tank VCO Architektur (vgl. Abb. 1) beschränkt. Es lässt sich auch leicht auf komplexere Verschaltungen von MOS Varaktoren anwenden. Ein Beispiel für eine 


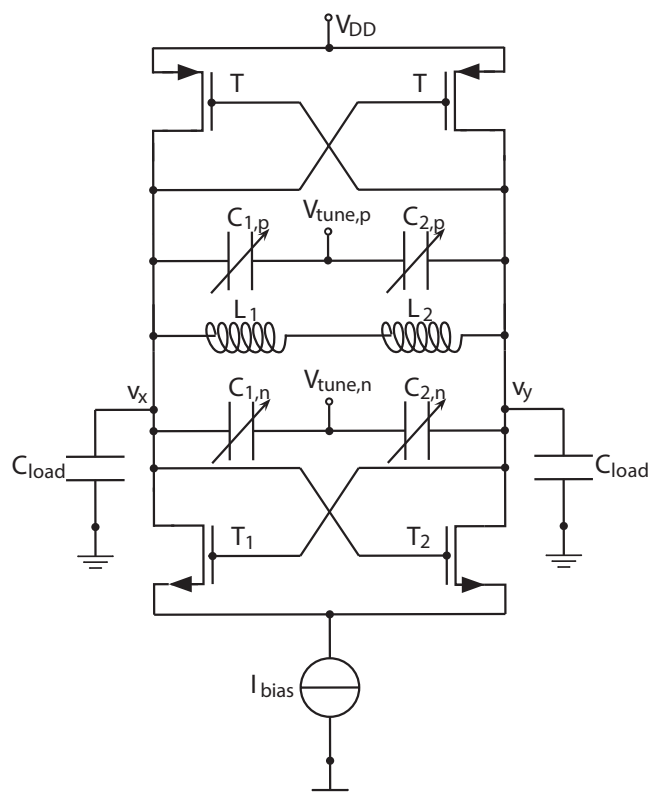

Abb. 8. LC-Tank VCO mit differentielle Verschaltung der Varaktoren.

komplexe Varaktorverschaltung stellt die in Abb. 8 gezeigte differentielle Varaktorverschaltung dar (Tiebout, 2001). Diese Verschaltungsart optimiert signifikant das AM-FM Konversionsverhalten des zugrunde liegenden VCOs (Tiebout, 2001), benötigt aber auch die doppelte Fläche im Vergleich zur back-to-back Verschaltung. Weitere Anwendungsmöglichkeiten des Simulationsmodells sind die weit verbreitete VCO Topologie mit zuschaltbaren festen Kapazitäten (Soltanian und Kinget, 2006) und die VCO Topologie bestehend aus einer Parallelschaltung mehrerer VCOs (Kral et al., 1998). Das in dem Computeralgebrasystem MAPLE implementierte Simulationsmodell ist auf eine Vielzahl von MOS Varaktor- und VCO-Topologien anwendbar.

\subsection{Effektive Großsignalkapazität}

In einer Standard CMOS LC-Tank Topologie liegt die Ausgangsamplitude direkt über den Gateanschlüssen der verwendeten MOS Varaktoren. Diese Eigenschaft führt dazu, dass sich die sich einstellende effektive Varaktorkapazität periodisch mit dem Ausgangssignal $v_{t}(t)$ des VCOs ändert. Daraus folgt, dass zur Berechnung der sich einstellenden Kapazität eine Großsignalanalyse erforderlich ist. Hierfür wurde in Bremer et al. (2009) eine Berechnung der Großsignalkapazität aus dem analytischen Ausdruck für die Kleinsignalkapazität (18) vorgestellt. In dem Ansatz wird die Annahme getroffen, dass durch den symmetrischen Aufbau der LC-Tank-Struktur, auch die MOS Varaktortransistoren vollständig symmetrisch sind. Daraus folgt für das differentielle Ausgangssignal des VCOs

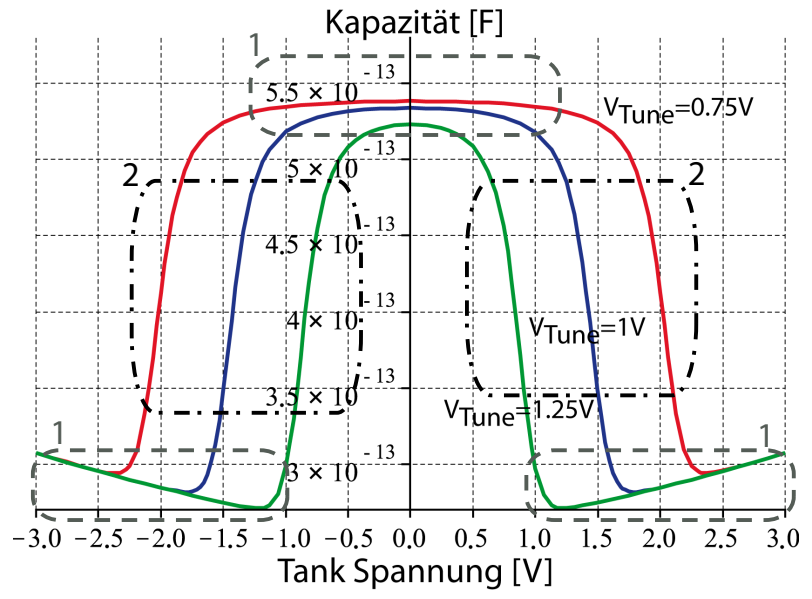

Abb. 9. Gesamtvaraktorkapazität eines NMOS Inversiosmode Varaktors mit einer Transistorweite von $W_{\mathrm{v}}=400 \mu \mathrm{m}$.

$v_{x}(t)-v_{y}(t)=\frac{v_{t}(t)}{2}-\frac{-v_{t}(t)}{2}$.

Damit ergibt sich für die ausgangssignalabhängige Großsignalkapazität eines LC-Tank VCOs mit back-to-back Varaktorverschaltung (vgl. Abb. 1) der folgende Ausdruck

$C_{\text {var }}\left(v_{t}, V_{\text {Tune }}\right)=\frac{C_{1}\left(\frac{v_{t}}{2}, V_{\text {Tune }}\right) \cdot C_{2}\left(-\frac{v_{t}}{2}, V_{\text {Tune }}\right)}{C_{1}\left(\frac{v_{t}}{2}, V_{\text {Tune }}\right)+C_{2}\left(-\frac{v_{t}}{2}, V_{\text {Tune }}\right)}$.

In Abb. 9 ist die CV-Charakteristik der Großsignalgesamtkapazität in Abhängigkeit des Ausgangssignals $v_{t}(t)$ und der Varaktorsteuerspannung $V_{\text {Tune }}$, für verschiedene Steuerspannungen dargestellt. In den mit 1 gekennzeichneten Bereichen der CV-Kurve besitzen die Varaktoren eine geringe Neigung $\mathrm{AM}$ in FM zu konvertieren. In den Bereichen, die mit 2 gekennzeichnet sind, besitzen die Varaktoren hingegen eine hohe Neigung AM in FM zu konvertieren.

\subsection{Systematischer VCO Entwurfsprozess}

Die steigenden Anforderungen die an VCO-Schaltungen gestellt werden, um die modernen Kommunikationsstandards zu erfüllen, führen zu immer komplexeren Schaltungsstrukturen, wie z.B. die differentielle Verschaltung der Varaktoren (vgl. Abb. 8). Durch diese, in ihrer Komplexität anwachsenden Schaltungsstrukturen, steigt der Aufwand und Zeitbedarf in der Entwurfsphase beachtlich an. Um den Aufwand $\mathrm{zu}$ reduzieren, wird ein analytischer Ansatz benötigt, mit dem im Vorfeld des Einsatzes eines professionellen CADTools die Entwurfsparameter des VCOs approximiert und optimiert werden können. In Bremer et al. (2009) wurde ein systematischer VCO Entwurfsprozess basierend auf der Andronov-Hopf-Bifurkationsanalyse und der Störungsrechnung vorgestellt. Auf Basis dieses systematischen Entwurfskonzepts wurde ein LC-Tank VCO mit einer Mittenfrequenz 


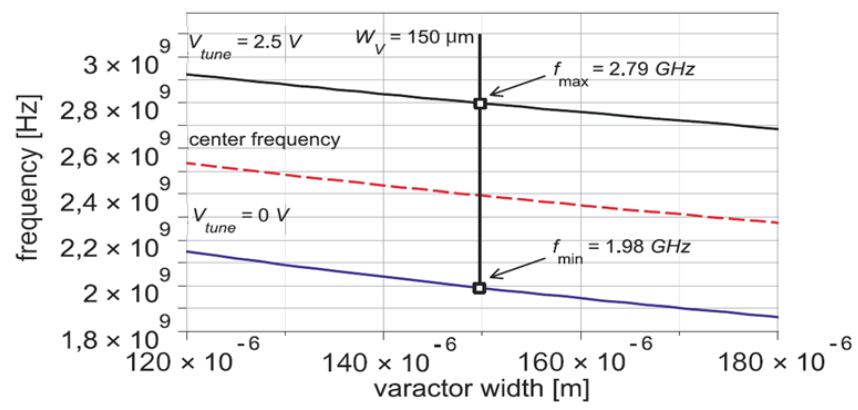

Abb. 10. Varaktor Dimensionierung basierend auf dem analytischen Simulationsmodell (siehe Bremer et al., 2009).

von $2,4 \mathrm{GHz}$, der die Bluetooth Spezifikationen erfüllt, entworfen. Wie die Abb. 10 verdeutlicht, ermöglicht eine Integration des analytischen Varaktorsimulationsmodells in den systematischen Entwurfsprozess eine Approximation der Varaktorlängen und -weiten, die die geforderten Spezifikationen erfüllen.

\subsection{AM-FM Optimierung}

In der LC-Tank VCO Architektur liegt die Ausgangsamplitude direkt über den Varaktoren an. Dies hat zur Folge, dass eine Modulation der Amplitude eine direkte Modulation der Ausgangsfrequenz mit sich führt. Um dieses störende Verhalten im Vorfeld zu optimieren, ist es sinnvoll einen AMFM Sensibilitätsfaktor für die Varaktoren einzuführen (Hegazi und Abidi, 2003)

$K_{\mathrm{AMFM}} \triangleq \frac{\partial \omega_{0}}{\partial A_{\mathrm{VCO}}}=-\frac{1}{2} \frac{\omega_{0}}{C_{\mathrm{eff}}} \frac{\partial C_{\mathrm{eff}}}{\partial A_{\mathrm{VCO}}}$.

Dieser Sensibilitätsfaktor ist durch die Differentiation der Oszillationsfrequenz nach der Amplitude definiert. In Hegazi und Abidi (2003) wird der Sensibilitätsfaktor berechnet, indem im Vorfeld die effektive Varaktorkapazität über ein grafisches Verfahren ermittelt wird. Ein alternatives Vorgehen zur Bestimmung des Sensibilitätsfaktors, das ohne grafisches Verfahren auskommt, basiert auf der Beschreibung der VCO-Schaltung durch ein Differentialgleichungssystem zweiter Ordnung der folgenden Form (Zorn et al., 2008)

$\left(\begin{array}{l}\frac{d v_{t}}{d t} \\ \frac{d i_{L}}{d t}\end{array}\right)=\left(\begin{array}{cc}\frac{1}{R_{t} C_{t}} & \frac{1}{C_{t}} \\ \frac{1}{L_{t}} & 0\end{array}\right)\left(\begin{array}{c}v_{t} \\ i_{L}\end{array}\right)+\left(\begin{array}{c}\frac{i_{d}\left(v_{t}\right)}{C_{t}} \\ 0\end{array}\right)$,

mit der Tankkapazität $C_{t}=C_{\mathrm{var}}\left(v_{t}, V_{\text {Tune }}\right)+C_{\mathrm{par}}$, die sich aus der Gesamtvaraktorkapazität $C_{\mathrm{var}}\left(v_{t}, V_{\text {Tune }}\right)$ aus (20) und den parasitären Kapazitäten des VCOs $C_{\text {par }}$ zusammensetzt. Aufgrund des analytischen Varaktorsimulationsmodells und der nichtlinearen Modellierung der einzelnen Schaltungskomponenten des VCOs ist das Differentialgleichungssystem (22) ausschließlich von Prozess- und Designparametern abhängig. Ausgehend von diesem nichtlinearen Differentialgleichungssystem ist es möglich, mittels Methoden der
Störungsrechnung, analytische Näherungen für die Frequenz und die Amplitude des VCOs zu bestimmen (Mathis, 1987). Die für dieses Vorgehen geeignete Methoden der Störungsrechnung sind z.B. die Methode nach Poincaré-Linsted, die Mittelungsmethode oder die Methode der vielfachen Zeitskalen (Nayfeh, 1993). Mit der aus der Störungsrechnung erhaltenen Näherung für die Amplitude und die Frequenz lässt sich der Sensibilitätsfaktor aus (21) direkt bestimmen. Auf Basis des so gewonnen analytischen Ausdrucks des AM-FM Sensibilitätsfaktors lassen sich die Varaktoren während des Schaltungsentwurfs hinsichtlich des AM-FM Verhaltens und hiermit die Phasenrauscheigenschaften des VCOs optimieren.

\section{Diskussion}

Es wurde ein analytisches Simulationsmodell für Inversionsmode-MOS-Varaktoren vorgestellt. Dieses Modell basiert ausschließlich auf Prozess- und Designparametern und benötigt keine empirischen Faktoren, die im Vorfeld durch Simulation oder durch Messungen bestimmt werden müssen. Die Gültigkeit und Genauigkeit des Simulationsmodells wurde anhand von Spectre (Cadence) Simulationen auf Basis eines $0.25 \mu \mathrm{m}$ CMOS Prozesses der Firma IHP und eines $0.35 \mu \mathrm{m}$ CMOS Prozesses der Firma AMS verifiziert. Die Simulationsergebnisse zeigten in allen Transistorbetriebsbereichen, sowohl für NMOS als auch PMOS Varaktoren, eine gute Übereinstimmung der CV-Charakteristik mit den Spectre (Cadence) Simulationsergebnissen. Abschließend wurden vielfältige Anwendungsmöglichkeiten für das Varaktorsimulationsmodell aufgezeigt, wie z.B. die Berechnung der effektiven Großsignalkapazität des VCOs, die Dimensionierung der Varaktoren in einem systematischen LC-Tank VCO Entwurfsprozess oder die Optimierung des AM-FM Verhaltens der Varaktoren im VCO.

\section{Literatur}

Buonomo, A.: Nonlinear Analysis of Voltage-Controlled Oscillators: A Systematic Approach, Circuits and System I: Regular Papers, IEEE Transactions, 55(6), 1659-1670, 2008.

Bremer, J., Zorn, C., Przytarski, J., und Mathis, W.: A Nonlinear Systematic Design Flow for LC Tank VCOs Based on Large Signal Capacitance Modeling, Proceedings of the IEEE International Symposium on Circuits and Systems ISCAS, Taipei, Taiwan, 2389-2392, 2009.

Bucher, M., Lallement, C., und Enz, C.: An Efficient Parameter Extraction Methodology for the EKV MOST Model, IEEE Int. Conference on Microelectronic Test Structures, 9, 145-150, 1996.

Bucher, M., Lallement, C., Enz, C., Theodoloz, F., und Krummenacher, F.: The EPFL-EKV 2.6 MOSFET Model Equations for Simulation, Technical Report, EPFL, http://legwww.epfl.ch/ ekv/, 1997. 
Enz, C.-C. und Vittoz, E.-A.: Charge-Based MOS Transistor Modeling: The EKV Model for Low-Power and RF IC Design, Wiley, 2006.

Hegazi, E. und Abidi, A. A.: Varactor Characteristics, Oscillator Tuning Curves and AM-FM Conversion, IEEE J. Solid-St. Circ., 38(6), 1033-1039, 2003.

Hershenson, M., Hajimiri, A., Mohan, S., Boyd, S., und Lee, T.: Design and optimization of LC oscillators, Proceedings IEEE/ACM international conference on Computer-aided design, 65-69, 1999.

Kral, A., Behbahani, F., und Abidi, A. A.: RF-CMOS Oszillators with switched tuning, Custom Integrated Circuits Conference, IEEE, 555-558, 1998.

Mathis, W.: Theorie nichtlinearer Netzwerke, Springer, 1987.

Nayfeh, A.: Introduction to Perturbation Techniques, Wiley-VCH, 1993.
Soltanian, B. und Kinget, P.: AM-FM conversion by the active devices in MOS LC-VCOs and its effect on the optimal amplitude, Radio Frequency Integrated Circuits RFIC Symposium, IEEE, 104-108, 2006.

Stefanovic, D. und Kaya, M.: Structured Analog CMOS Design, Springer Publishung Company, Incorporated, 2008.

Tiebout, M.: Low-Power Low-Phase-Noise differentially tuned Quadrature VCO Design in Standard CMOS. Solid-State Circuits, IEEE Journal, 36(7), 1018-1024, 2001.

Zorn, C., Bremer, J.-K., und Mathis, W.: Ansätze für eine Bifurkationsanalyse von RF LC-Tank VCOs unter Berücksichtigung nichtlinearer Bauelementegleichungen, Adv. Radio Sci., 6, 189194, doi:10.5194/ars-6-189-2008, 2008. 